\title{
A Trilogy of Influenza, Immunization, and Antimicrobial Resistance: A Review Article
}

\author{
Ilad Alavi Darazam (iD) ${ }^{1,2,{ }^{*}}$ and Masoud Mardani (iD) ${ }^{1,2}$ \\ ${ }^{1}$ Infectious Diseases and Tropical Medicine Research Center, Shahid Beheshti University of Medical Sciences, Tehran, Iran \\ ${ }^{2}$ Department of Infectious Diseases and Tropical Medicine, Loghman Hakim Hospital, Shahid Beheshti University of Medical Sciences, Tehran, Iran \\ "Corresponding author: Infectious Diseases and Tropical Medicine Research Center, Shahid Beheshti University of Medical Sciences, Tehran, Iran. Email: ilad13@yahoo.com
}

Received 2019 January 27; Accepted 2019 February 17.

\begin{abstract}
Influenza virus could lead in a mild acute respiratory illness or severe systemic and respiratory disease, therefore, during the activity of influenza considerable number of respiratory syndromes and complications can be related to this virus. On the other hand, risk for concomitant and post-influenza bacterial complications threat is not huge enough to consider to prescribe empiric antibiotics. Influenza vaccine prevents millions of illnesses and flu-related visits every year. Influenza immunization has clear effects on disease burden as well as the number of patients with resistant disease acquired and transmitted in communities. Vaccination program indirectly diminishes antibiotic use and reduces the exposure of bacteria to antibiotics and consequently selective pressure for resistance. It seems there is definite evidence to consider annual influenza immunization in antimicrobial stewardship program and promote the global and national health quality. Hereby, we review scientific evidence of direct and indirect effects of influenza as well as the influence of vaccination and prevention program health systems.
\end{abstract}

Keywords: Antimicrobial Resistance, Influenza, Vaccine, Antibiotics

\section{Antimicrobial Resistance}

Global statistics in 2014, even though with an underestimation, disclosed about 700,000 annual mortality from drug-resistant strains of common bacterial infections, HIV, $\mathrm{TB}$, and malaria (1).

Resistance to at least first-line antimicrobials in the United States, nearly two million per year, costs an extra $\$ 20$ billion to the health system (2). Assuming the drug resistance prevalence of six main microorganisms by 2050 , the mortality rate could reach one person every three seconds (1).

It is clear that antibiotic resistance can lead to prolonged hospitalization, higher medical charges, and increased death. More antibiotics consumption directly causes more drug resistance. Hence, focusing on justified antimicrobial prescription can strongly influence resistance.

Up to May 2017, 42 new traditional antibiotics and biological agents were developed to target critical and high-priority pathogens in the clinical pipeline. Although they are promising treatments, the clinical development pipeline solely is inadequate to combat the rising resistance. Effective antimicrobials are insufficient for the main resistant bacteria, particularly multidrug and extensively drug-resistant Gram-negative pathogens. Antibiotic development must go hand in hand with attempts to promote the appropriate use of current and future antibiotics (3).

Therefore, the burden of antimicrobial resistance (AMR) as a global, political, and economic issue led to the political declaration of the high-level meeting of the general assembly on antimicrobial resistance in 2016 in United Nations (UN). It declares “... underline further that affordability and access to existing and new antimicrobial medicines, vaccines and diagnostics should be a global priority and should take into account the needs of all countries, in line with the World Health Organization global strategy and plan of action on public health, innovation and intellectual property, and taking into consideration its internationally agreed follow-up processes" (4).

\section{Antimicrobial Over-Prescription}

Overdiagnosis and antimicrobials over-prescription are major global and national problems all around the world including regions with the high-level socioeconomic condition. The White House National Action Plan for Combating Antibiotic-Resistant Bacteria established a 
national aim to reduce outpatient antibiotic prescription by $50 \%$ by 2020 .

In $2010-2011,12.6 \%$ of the 184032 outpatient visits in the USA were accompanied with antibiotic prescriptions, with an about 506 antibiotic prescriptions per 1000 USA population annually. It was also shown that acute respiratory conditions were associated with approximately 221 antibiotic prescriptions per 1000 population every year. The researchers supposed that considerable antibiotic overprescription is likely driven from overdiagnosis of certain acute respiratory conditions, such as sinusitis diagnosis without meeting criteria (5).

Another national study concerning emergency departments in the USA showed that, between 2001 and 2010, there were 126 million visits with a diagnosis of acute respiratory tract illness (ARI) and excessive rate of antibiotics prescription (about 61\%). While major improvement has been made toward the reduction of antibiotic consumption for children with ARI, the proportion of adults with the same conditions receiving antibiotics in emergency departments was extraordinary high (6).

In Nordic countries, the majority of the patients were prescribed with antibiotics with a small number of appropriate prescriptions. In general, sinusitis and pharyngotonsillitis were respiratory illnesses with an inappropriate antibiotic prescription (7).

In the Middle East, reports on antibiotics consumption are not promising. A prospective cross-sectional study in outpatient care clinics in Jordan showed that antibiotics were prescribed for $78.4 \%$ of patients aged less than 18 years presenting with ARI while antibiotics were not indicated in $69.2 \%$. Furthermore, broad-spectrum antibiotics were prescribed in $51.1 \%$ of all antibiotic-prescribed participants (8).

Similar results from other studies demonstrate that most UK general practices ordered antibiotics to young and middle-aged adults with ARI at proportions significantly higher than what was clinically necessary, leading to antibiotic resistance (9).

In the Netherlands, over-prescription of antibiotics was highest for patients between 18 and 65 years of age, when general physicians felt patients' pressure for antibiotic treatment, and for patients presenting with fever and complaints of longer than one week (10).

These data and many others from all over the world show that over-prescription of antibiotics is a global issue and it is not restricted to a few countries. Moreover, statistics demonstrate that ARI takes the highest rate of inappropriate antibiotic consumption. As mentioned above, there is a direct correlation between antibiotics consumption and the AMR rate. Thus, concentrating on reducing this ratio for ARI could be a central goal for controlling AMR.

\section{Influenza and Antibiotics}

Influenza has a broad spectrum of non-significant symptoms from a mild ARI to severe systemic and respiratory disease. Thus, during influenza activity, a considerable number of ARIs and other complications could be related to influenza.

A study showed that the consumption of respiratory fluoroquinolone is seasonal and strongly associated with influenza activity (20\% versus $8 \%$ ) (11). There is usually a concern about bacterial superinfection or coinfection with influenza but studies demonstrate that a moderate number of patients develop a bacterial infection following an episode of influenza $(11,12)$. Although physicians should think about possible bacterial coinfections in all influenza cases, they should not assume all patients are coinfected. The authors explained that because antibiotics are not effective against viral infections and the antimicrobials are not useful in preventing bacterial superinfections, a substantial number of antimicrobial treatments during influenza outbreaks may be redundant and probably contribute to antimicrobial resistance (11).

Neuzil et al. showed that influenza activity directly influenced the number of hospital admissions for cardiopulmonary conditions in excess of the expected numbers among children (13). This study showed that for every 100 children, a yearly average of 6 to 15 outpatient visits and 3 to 9 courses of antibiotics were attributable to influenza. They concluded that $10 \%$ to $30 \%$ of the additional number of antibiotic courses occurred during influenza activity (13).

A considerable percentage of Korean children (81\%) with influenza received antibiotic monotherapy while only $4 \%$ of the patients received oseltamivir alone. The researchers demonstrated that the average charges for hospitalizations with antibiotics treatment, alone or with oseltamivir, were meaningfully more than the charges of hospitalizations treated with oseltamivir-only. Interestingly, influenza patients treated with antibiotics-only and antibiotics/oseltamivir had a longer duration of hospitalization than those treated with oseltamivir-only (14).

In another study, pneumonia among elderly and adult patients with underlying risk factors taking placebo was $2.5 \%$ (crude number of pneumonia cases without treatment). In this study, even though with small size, neuraminidase inhibitors seemed to be protective. Comparable findings in other studies showed a $1.6 \%$ rate of bacterial complications of influenza. Overall, available data propose that during regular influenza activity, around $1 \%$ of the patients with influenza will develop secondary bacterial pneumonia (15). These findings disclose that even though there is a risk of bacterial complications, this threat is not 
huge enough to convince clinicians to use empiric antibiotics.

\section{Influenza Vaccination and Antimicrobial Resis- tance}

Vaccines can, directly and indirectly, influence antimicrobial resistance. Direct effects include diminishing disease burden and the number of patients with resistant disease acquired and transmitted in the population. Indirect effects include decreased antibiotic use. The latter effect reduces the exposure of bacteria to antibiotics and consequently selective pressure for resistance $(16,17)$. A common case of this type of prescription would be the use of antibiotics for influenza.

In a population-based study with 33137 children treated with 378 general practices during the consecutive years, $43.4 \%$ with at least one amoxicillin prescription had been vaccinated. The proportion of amoxicillin consumption significantly decreased during the periods of influenza vaccine immunity. The researchers demonstrated that amoxicillin use could have been reduced by 5.6\% if the immunization rate in children increased to 50\%. Therefore, they suggested influenza vaccination of young children could contribute to diminished amoxicillin consumption, one of the most common antibiotics (18). In another study, influenza vaccine significantly reduced acute otitis media episodes and related complications among children (19).

Herd immunity describes vaccinated individuals who do not themselves become infected or colonized, and hereafter they do not transmit the pathogen to the community. Therefore, infections, resistant infections, and antimicrobial expenditure can be reduced among vaccinated persons and in their contacts including groups of individuals for whom the vaccine is not licensed, those that cannot be immunized, or immunocompromised hosts $(20,21)$.

In a survey, the effectiveness of influenza vaccine was evaluated among mothers and their neonates. The researchers demonstrated that maternal influenza vaccination was significantly associated with a low likelihood of febrile episodes and ARIs in infants and antibiotics use during the influenza season. They concluded that postpartum influenza vaccination of mothers was associated with a substantial reduction of influenza-related complications, healthcare seeking, and antibiotic consumption in infants (22).

Influenza vaccine prevents millions of illnesses and flurelated visits annually. During 2016 - 2017, it prevented approximately 5.3 million influenza illnesses, 2.6 million influenza-related visits, and 85,000 influenza-associated hospitalizations. When the vaccine contains viruses with matched circulating strains, the vaccine shows to decrease the risk of medical visits by $40 \%$ to $60 \%$. On the other hand, vaccination can reduce the risk of hospitalization due to influenza for all age groups. During 2016 - 2017, immunization against influenza prevented around 85,000 influenzaassociated hospitalizations (23).

Eventually, a Canadian study confirmed that the rate at which influenza-associated antibiotics were prescribed was $\sim 64 \%$ lower after the use of universal influenza immunization in Ontario than other regions that restricted the use of influenza vaccines to at-risk situations (24).

\section{Conclusion}

Antimicrobial resistance as a major ongoing problem in the world requires meticulous considerations. Antibiotic consumption rate must be reduced. One of the most important and practical factors influencing antibiotics prescription rate is containing acute respiratory illnesses. Influenza during activity is a major problem that directly and indirectly increases the antibiotics consumption rate. Therefore, it seems preventing influenza and influenzarelated complications eventually reduces antibiotics prescription, as well as the development of antimicrobial resistance. Immunization is an effective approach to protect individuals against influenza. Accordingly, influenza vaccination could be a safe and effective program to reduce AMR. However, the reliable quantification of reduced AMR development after widespread influenza vaccination is challenging as several factors can considerably influence vaccine effectiveness, and the use of influenza vaccines by itself is debated.

\section{Footnotes}

Authors' Contribution: Both authors have contributed equally in preparing all parts of the article.

\section{Conflict of Interests: None.}

Ethical Considerations: This review article has not any ethical consideration.

Funding/Support: None.

\section{References}

1. O’Neill J. Tackling drug-resistant infections globally: Final report and recommendations: The review on antimicrobial resistance. London; 2016.

2. Golkar Z, Bagasra O, Pace DG. Bacteriophage therapy: A potential solution for the antibiotic resistance crisis. JInfect Dev Ctries. 2014;8(2):12936. doi: 10.3855/jidc.3573. [PubMed: 24518621].

3. World Health Organization (WHO). Antibacterial agents in clinical development: An analysis of the antibacterial clinical development pipeline, including tuberculosis. Geneva; 2017.

4. United Nations (UN). Draft political declaration of the high-level meeting of the general assembly on antimicrobial resistance. New York: UN; 2016. 
5. Fleming-Dutra KE, Hersh AL, Shapiro DJ, Bartoces M, Enns EA, File TM $\mathrm{Jr}$, et al. Prevalence of inappropriate antibiotic prescriptions among us ambulatory care visits, 2010-2011. JAMA. 2016;315(17):1864-73. doi: 10.1001/jama.2016.4151. [PubMed: 27139059].

6. Donnelly JP, Baddley JW, Wang HE. Antibiotic utilization for acute respiratory tract infections in U.S. emergency departments. Antimicrob Agents Chemother. 2014;58(3):1451-7. doi: 10.1128/AAC.02039-13. [PubMed: 24342652]. [PubMed Central: PMC3957838].

7. Run Sigurethardottir N, Nielsen AB, Munck A, Bjerrum L. Appropriateness of antibiotic prescribing for upper respiratory tract infections in general practice: Comparison between Denmark and Iceland. Scand J Prim Health Care. 2015;33(4):269-74. doi: 10.3109/02813432.2015.1114349. [PubMed: 26683287]. [PubMed Central: PMC4750736].

8. Ababneh MA, Al-Azzam SI, Ababneh R, Rababa'h AM, Demour SA Antibiotic prescribing for acute respiratory infections in children in Jordan. Int Health. 2017;9(2):124-30. doi: 10.1093/inthealth/ihx003. [PubMed: 28338813].

9. Gulliford MC, Dregan A, Moore MV, Ashworth M, Staa T, McCann $\mathrm{G}$, et al. Continued high rates of antibiotic prescribing to adults with respiratory tract infection: Survey of 568 UK general practices. BMJ Open. 2014;4(10). e006245. doi: 10.1136/bmjopen-2014-006245. [PubMed: 25348424]. [PubMed Central: PMC4212213].

10. Dekker AR, Verheij TJ, van der Velden AW. Inappropriate antibiotic prescription for respiratory tract indications: Most prominent in adult patients. Fam Pract. 2015;32(4):401-7. doi: 10.1093/fampra/cmv019. [PubMed: 25911505].

11. Polgreen PM, Yang M, Laxminarayan R, Cavanaugh JE. Respiratory fluoroquinolone use and influenza. Infect Control Hosp Epidemiol. 2011;32(7):706-9. doi: 10.1086/660859. [PubMed: 21666403]. [PubMed Central: PMC3258490].

12. Klein EY, Monteforte B, Gupta A, Jiang W, May L, Hsieh YH, et al. The frequency of influenza and bacterial coinfection: A systematic review and meta-analysis. Influenza Other Respir Viruses. 2016;10(5):394403. doi: 10.1111/irv.12398. [PubMed: 27232677]. [PubMed Central: PMC4947938].

13. Neuzil KM, Mellen BG, Wright PF, Mitchel EF Jr, Griffin MR. The effect of influenza on hospitalizations, outpatient visits, and courses of antibiotics in children. $N$ Engl J Med. 2000;342(4):225-31. doi: 10.1056/NEJM200001273420401. [PubMed: 10648763].

14. Lim JK, Kim TH, Kilgore PE, Aiello AE, Choi BM, Lee KC, et al. The association between influenza treatment and hospitalizationassociated outcomes among Korean children with laboratoryconfirmed influenza. J Korean Med Sci. 2014;29(4):485-93. doi: 10.3346/jkms.2014.29.4.485. [PubMed: 24753694]. [PubMed Central: PMC3991790].

15. Metersky ML, Masterton RG, Lode H, File TM Jr, Babinchak T. Epidemiology, microbiology, and treatment considerations for bacterial pneumonia complicating influenza. Int J Infect Dis. 2012;16(5):e321-31. doi: 10.1016/j.ijid.2012.01.003. [PubMed: 22387143].

16. Atkins KE, Lipsitch M. Can antibiotic resistance be reduced by vaccinating against respiratory disease? Lancet Respir Med. 2018;6(11):8201. doi:10.1016/S2213-2600(18)30328-X. [PubMed: 30076121].

17. Wilby KJ, Werry D. A review of the effect of immunization programs on antimicrobial utilization. Vaccine. 2012;30(46):6509-14. doi: 10.1016/j.vaccine.2012.08.031. [PubMed: 22940379].

18. Hardelid P, Ghebremichael-Weldeselassie Y, Whitaker H, Rait G, Gilbert R, Petersen I. Effectiveness of live attenuated influenza vaccine in preventing amoxicillin prescribing in preschool children: A selfcontrolled case series study. J Antimicrob Chemother. 2018;73(3):779-86. doi: 10.1093/jac/dkx463. [PubMed: 29228207].

19. Marchisio P, Esposito S, Bianchini S, Dusi E, Fusi M, Nazzari E, et al. Efficacy of injectable trivalent virosomal-adjuvanted inactivated in fluenza vaccine in preventing acute otitis media in children with recurrent complicated or noncomplicated acute otitis media. Pediatr Infect Dis J. 2009;28(10):855-9. doi: 10.1097/INF.0b013e3181a487b4. [PubMed: 19564812].

20. Lipsitch M, Siber GR. How can vaccines contribute to solving the antimicrobial resistance problem? MBio. 2016;7(3). doi: 10.1128/mBio.00428-16. [PubMed: 27273824]. [PubMed Central: PMC4959668]

21. Jansen KU, Knirsch C, Anderson AS. The role of vaccines in preventing bacterial antimicrobial resistance. Nat Med. 2018;24(1):10-9. doi: 10.1038/nm.4465. [PubMed: 29315295].

22. Maltezou HC, Fotiou A, Antonakopoulos N, Kallogriopoulou C, Katerelos P, Dimopoulou A, et al. Impact of postpartum influenza vaccination of mothers and household contacts in preventing febrile episodes, influenza-like illness, healthcare seeking, and administration of antibiotics in young infants during the 2012-2013 influenza season. Clin Infect Dis. 2013;57(11):1520-6. doi: 10.1093/cid/cit599. [PubMed: 24046313].

23. Centers for Disease Control and Prevention (CDC). CDC reports on vaccine benefits from 2016-2017 season. Atlanta: CDC; 2018.

24. Kwong JC, Maaten S, Upshur RE, Patrick DM, Marra F. The effect of universal influenza immunization on antibiotic prescriptions: An ecological study. Clin Infect Dis. 2009;49(5):750-6. doi: 10.1086/605087. [PubMed: 19624280]. 